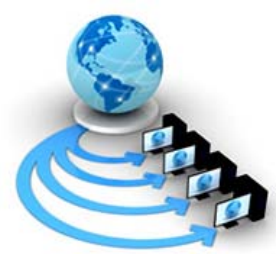

International Journal of Advanced Research in Computer Science

RESEARCH PAPER

\author{
Available Online at www.ijarcs.info
}

\title{
INCORPORATING BIOMETRIC AUTHENTICATION MECHANISM IN THE VOTING SYSTEM
}

\author{
A. Jenita Mary \\ Asst. Professor, \\ Department of Computer Applications \\ Faculty of Science and Humanities \\ SRM Institute of Science and Technology \\ Kattankulathur, Chennai
}

\author{
A.Subashini \\ Asst. Professor, \\ Department of Computer Applications \\ Faculty of Science and Humanities \\ SRM Institute of Science and Technology \\ Kattankulathur, Chennai
}

\begin{abstract}
An online voting system is to avoid multiple polling and irregularities at counting location. Currently, the voting system in India is inefficient and vulnerable to outer threats. The security check is depending on the voter Identity Card (ID) and the card is duplicated for an instance with varying addresses. Vote counting is done manually and is found that it is a process that consumes a big span of time. Due to the lack of securities in the rural areas the polling booths are captured and the ballots are destroyed. In order to avoid these kinds of disturbances, the implementation of an online voting system with digital securities and reusing the existing devices may incur less cost. This study proposes a Fingerprint Based Voting System, which uses a recognition part of the user through a fingerprint verification module. Since the fingerprint pattern is unique for humans, the authentication is done easily and duplicating such pattern is highly impossible. The system allows the voter to login through the fingerprint. The proposed method restricts the voter not to vote for more than one time.
\end{abstract}

Keywords: Fingerprint sensor, Fingerprint scanner, Touch screen, Biometrics, Online voting.

\section{INTRODUCTION}

A new voting system can be implemented using login which requires a finger print scan. It is a web application that supports irrespective of all browsers. Valid voters will have their name, fingerprint, and other citizenship (Aadhar) details in the government database. Therefore, a fingerprint scanner will ensure casting of votes by legitimate voters. A simple, user friendly interface is used to interact with the users and hence it could be a great help for the illiterate voters too. The focus is more on the visual representation of data, and no unnecessary links are used, the interface is made as simple as possible with only basic functionalities. Voting schemes have evolved from counting hands in early days to systems that include paper, punch card, mechanical lever and optical-scan machines.

In spite of the said, an electronic voting system which is used nowadays provide some features different from the traditional voting techniques, and also it provides improved features of voting over traditional voting system such as accuracy, convenience, flexibility, privacy, verifiability and mobility and so on, where as the present electronic voting systems suffers from drawbacks, which include consumption of time, large volume of paper work, lack of direct roles for the higher officials, damage of machines due to lack of attention; also updates from the user side like change of address is not done easily[1]. The system ensures the live status of the voter by registering an OTP which could be sent to the user and the same has to updated to the Aadhar details a few days before voting begins.

\section{PROPOSED SYSTEM}

In this study, we proposed a secure biometric based touch screen electronic voting system and it helps the user to cast vote from anywhere part of the globe. In general, user authentication is done through the classical username, password method[2]. The method of username, password is found lagging due the next big process that is to be continued when the password is forgotten.

The proposed system is incorporated with the biometric approaches to overcome such difficulties. Biometric system is a combination of methods that help us to identify people according to their physical characteristics. The biological input parameters may be considered from the tuple space that may consists of finger print, face, hand shape, retina, iris and voice tracks[3]. These parameters may be used as single or in a combinatorial form. Finger print recognition system is the leading method because it may not change over course of time and is easy to use; also possibility of losing, forgetting and stealing is impossible.

In this research work, fingerprint authentication method is used a part of voting system to avoid the difficulties faced by the government. The Administration Role (admin) is allowed to add name, photo and fingerprint of a candidate who is nominated for any post in the election. Admin will register the voters to an enrollment list to be used for the purpose by taking the needed information of a citizen from the existing database, the Aadhar. The age of a citizen will be derived from the person's date of birth and hence the age eligibility to vote is notified to the Aadhar database and updated. It is not needed to get enrolled to the voters list through a manual mode. The voter enrollment list will be garbage collected once the election is over. The life of the list of voters and the nominees will be five days prior 
to the election and a time suggested by the authority after the date of election.

The life status of the citizen is ensure through a One Time Password/Pin number(OTP), which will be sent by the election commission to the citizens and the same has to be verified through the combination of fingerprint and the OTP. This verification system may be encouraged through a mobile application; the inputs ensure the life status of the citizen. A return message to the registered mobile phone ensures the voter's registration and verification. This mobile application can be customized to the use of a personal mobile phone; also it can be used in a device under the control of a local authority of the panchayat or an officer in the civic body. The system allows the user to vote for an election and the same method of registering and getting verified shall be followed for any number of elections to come. The casted vote information shall be encrypted and shall be kept highly confidential. Fig. 1 through Fig. 5 illustrated the compilation of the proposed system.

\section{WORKING PRINCIPLE}

The Working Principle is shown below,

1. Scan the finger.

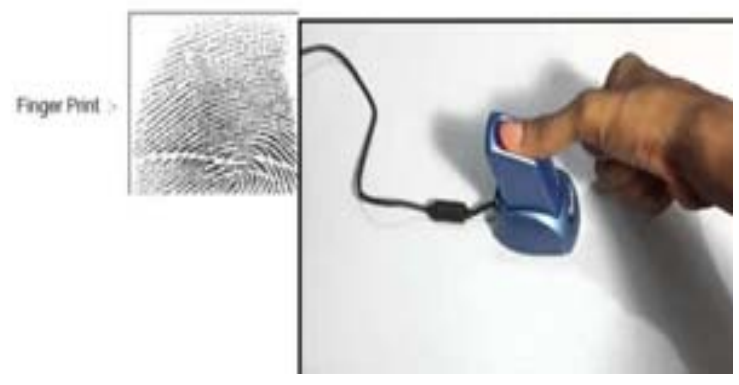

Fig. 1 Finger Print scanner

2. Finds match from the Aadhar Database and retrieve the voter information.

3. Ballot screen will be popped on the touch screen to cast the vote.

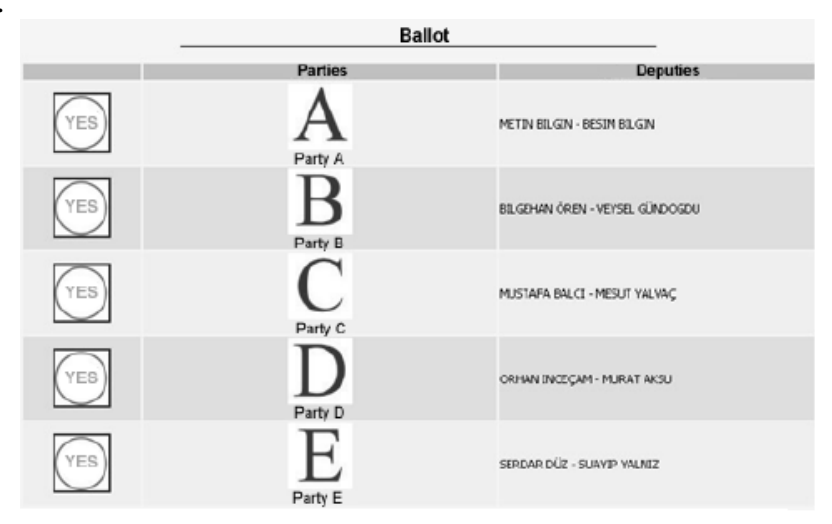

Fig. 2 Sample Ballot Screen

4. Auto-logout feature takes care of the rest after a vote has been placed and the main login screen is restored.
5. Once the vote is casted and name will be removed from the voters list.

The system ensures that no information should get stored in the host computer. The User Interface Unit shall consist of a display device, Subscriber Identity Module (SIM) card and a processor with a buffer of small capacity. The data get stored in the local machine shall be updated in a stipulated period of time and therefore the buffer shall be cleared. This unit can be taken to any part of the country without any specific requirements needed for installation.
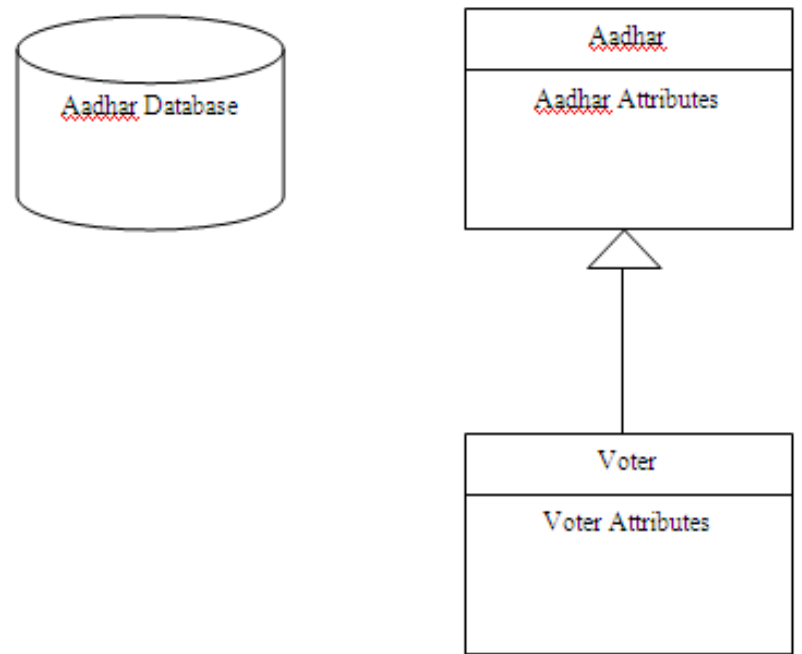

Fig. 3 Inherited Voters Enrollment List from Aadhar Database 


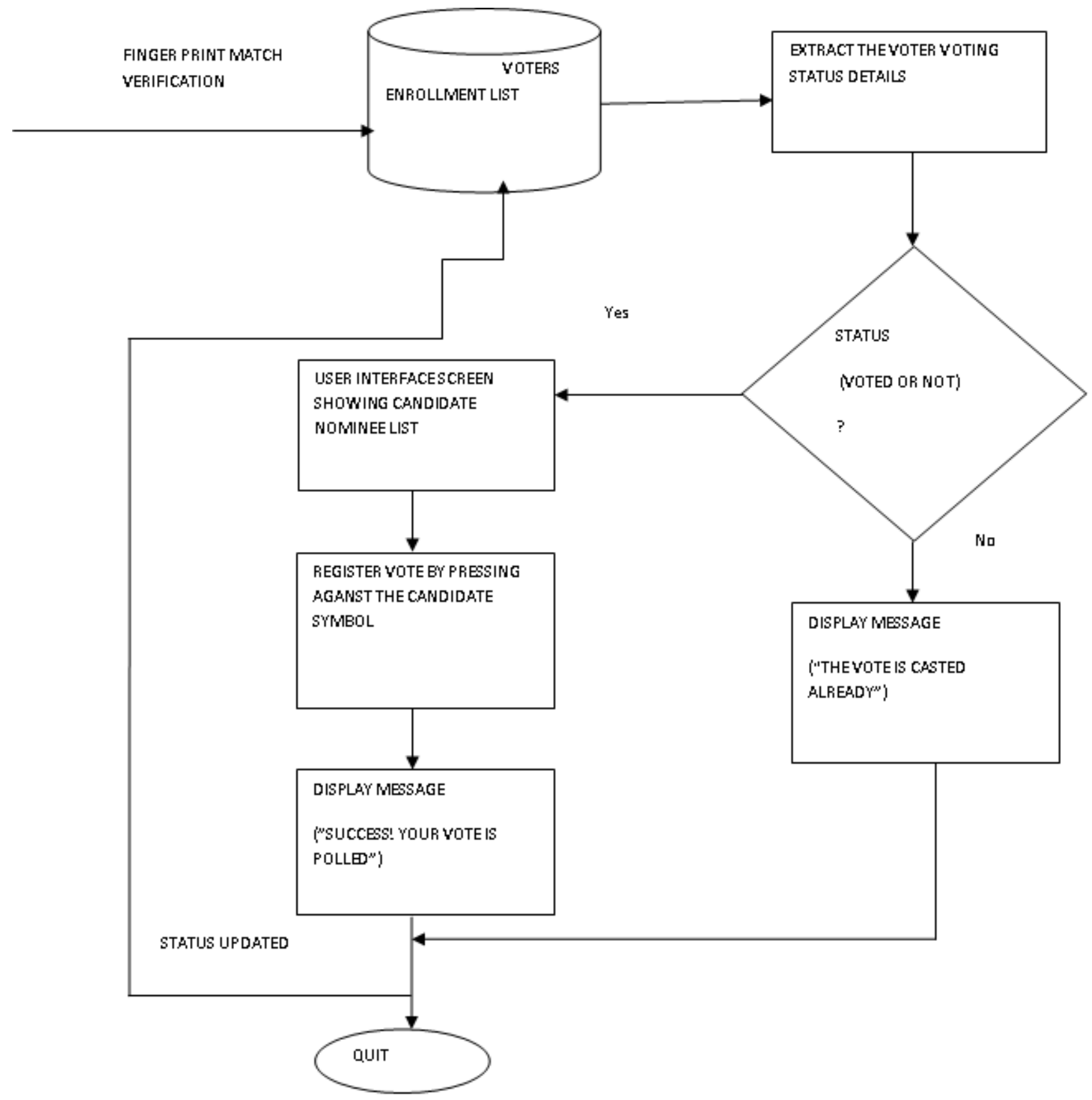

Fig. 4 work flow diagram

\section{CONCLUSION}

The proposed e-voting system is a user friendly method to the voters and is observed to find solutions for the prevailing civic problems. It is carefully planned to answer the issues faced by the rural citizens. Transporting the devices and getting internetwork connection is a notable serious issue in the present system; the implementation of the proposed solution shall overcome the same. It will be a better and faster method than the present system of voting, which has its own holes to the intruders who find possible way to make the voting system an illogical.

\section{REFERENCES}

[1] Rakesh S Raj ,Raghavendra A,Madhushree K R,Bhargavi D,“An Online Voting System Using Biometric Fingerprint and Aadhaar Card”, IJCAT International Journal of Computing and Technology, Vol. 1(4),2014,pp.87-92.

[2] Adem Alpaslan ALTUN ,Metin Bilgin , "Web based secure evoting system with fingerprint Authentication”, Scientific Research and Essays, Vol. 6(12),2011, pp. 2494-2500.

[3] M.Sudhakar, B.Divya Soundarya Sai, "Biometric System Based Electronic Voting Machine Using Arm9 Microcontroller” ,IOSR Journal of Electronics and Communication Engineering,Vol.10(1), 2015,pp. 57-65. 